\title{
Carl Sagan’s Conjecture of a Message in $\pi$
}

\author{
Arne Bergstrom* \\ B\&E Scientific Ltd, Seaford BN25 4PA, United Kingdom \\ *Corresponding author: arne.bergstrom@physics.org \\ Received November 04, 2014; Revised November 17, 2014; Accepted November 23, 2014
}

\begin{abstract}
In his novel Contact, the astrophysicist Carl Sagan hypothesized an alien message to be buried somewhere deep inside the numerical representation of the transcendental number $\pi$. The present article looks for markers that might possibly support such a hypothesis, and surprisingly finds a sequence of seven successive zeros (actually seven successive nines rounded off) at a depth of $3 \times 256$ digits into the representation of $2 \pi$ in the special case of base ten. Finding such a sequence of zeros within the first 1000 digits has a probability of 1 in 10000 . No such occurrences happen even remotely for $2 \pi$ at any base other than ten, nor even remotely in corresponding representations of other common transcendental numbers, such as $e$, which appear in physical applications. In $\pi$, this occurrence thus also remarkably appears at a depth that is a multiple of the same power of two as bits in a computer byte, which thus makes it even more enigmatic. Still, these effects are most probably just numerical coincidences without physical relevance.
\end{abstract}

Keywords: transcendental number pi, integer bases, markers, anthropic occurrence

Cite This Article: Arne Bergstrom, “Carl Sagan's Conjecture of a Message in $\pi$.” International Journal of Physics, vol. 2, no. 6 (2014): 264-266. doi: 10.12691/ijp-2-6-9.

\section{Introduction}

In his novel "Contact" [1], the late astrophysicist Carl Sagan lets an alien race contact mankind by a complicated, coded radio message. One interesting feature in Sagan's novel is that he lets the alien race disclose in passing that a master race may have left a message hidden deep inside the transcendental numbers.

As will be discussed in the following, there does indeed exist a surprising marker in the decimal representation of $\pi$, indicating the hypothetical possibility that there might possibly perhaps indeed be such a message encoded in it.

By our definition of $\pi$, it would seem to be an objectively well-defined transcendental number. However, the way we define $\pi$ actually contains a specific human and subjective characteristic, namely the concept of an ideal, flat space. It is only in such a space that the quotient between the circumference and diameter of a circle becomes the number $\pi$ as we define it [2] - and also in the corresponding expressions for the inscribed area and the volume of a sphere. In elliptic geometry [3], where the Euclidean postulates are all valid except the parallel postulate, the corresponding quotient is less than $\pi$. In Bolyai-Lobachevski's hyperbolic geometry [4], where the Euclidean postulates are also all valid except for the parallel postulate, the corresponding quotient is greater than $\pi$.

It is by no means certain, nor obvious, that the physical world should be absolutely flat and that the parallel postulate thus should be strictly valid in the physical world around us. That notion is a human assumption, and - if nothing else - the peculiar marker here found to exist in the representation of $\pi$ in just base ten could then serve to remind us of this fact.

In this connection we must ask whether it is really $\pi$, the ratio between the circumference and the diameter of the circle, which is to be regarded as the most natural ratio. Since the circle is defined - and constructed by a compass - as the curve which lies at a constant distance $r$ from a centre point, it is actually the radius $r$ rather than the diameter $2 r$ that must be regarded as the natural quantity for the ratio. Thus the natural ratio in the definition is the circumference to the radius, not the circumference to the diameter, and it is this ratio $2 \pi$ that we will mainly consider in the following (however, the conclusions here are essentially the same for both definitions).

\section{Representations as Function of Base and Depth}

A message intended to be noticed would be expected to be accompanied by some kind of marker, attracting the attention of a potential viewer and giving him some idea of where to look, and possibly also some clues how to decipher the message. Presumably, the marker would be some kind of separator in the message and perhaps at the same time an integral part of the message. A long string of, say, zeros in the transcendental number would serve this purpose.

It would thus be interesting to scan the most common transcendental numbers occurring in theoretical physics for strings of identical digits that are of abnormal length statistically. The transcendental number studied could of course be represented in different bases (binary, pental, octal, decimal, hexadecimal, etc), so the search has to be 
done for all possible simple bases. On the other hand, a potential intelligence reading the message need not necessarily be expected to have access to powerful computers but may possibly have to do all calculations "by hand", so presumably the message should not be buried too deep inside the digits of the transcendental number used. At least some preview would be expected to be found at some relatively shallow level in the number.

If, for instance, for base B = 8 we find the string 5555, then the probability of finding the second digit 5 immediately after the first one is $1 / 8$. The probability of finding another digit 5 immediately after again is $1 / 8^{2}$, and of finding still another immediately after again is another factor of $1 / 8$ less. So the probability of finding a string of four identical digits in base 8 is $1 / 8^{3}$. On the other hand, if we are calculating the number to a depth of, say, 2000 digits, then we would expect to get on the average $2000 / 8^{3}$ $\approx 3.9$ occurrences of such a string of four identical digits in base $\mathrm{B}=8$.

A particularly interesting marker is a sequence containing the digit 0 - or possibly also the digit corresponding to a shift to 0 , $i$ e the digit B-1 (like 9 in base 10) since such a string might be rounded off to a string of zeros. We assign special significance to sequences involving digits 0 by noting that the probability for a sequence of this type is a further factor of B lower.

Figure 1 through 4 display results of searches for such strings of identical digits for different bases and for different depths of digits in the number. The quantity called odds gives the average number of independent trials required to produce the sequence of identical numbers found when the transcendental number is calculated with 2000 digits in the particular base.

As an illustrative example, Figure 1 shows the transcendental number $e$ calculated with successively more digits in different bases (by using Maple [5]) in order to look for occurrences of abnormally long strings of identical digits.

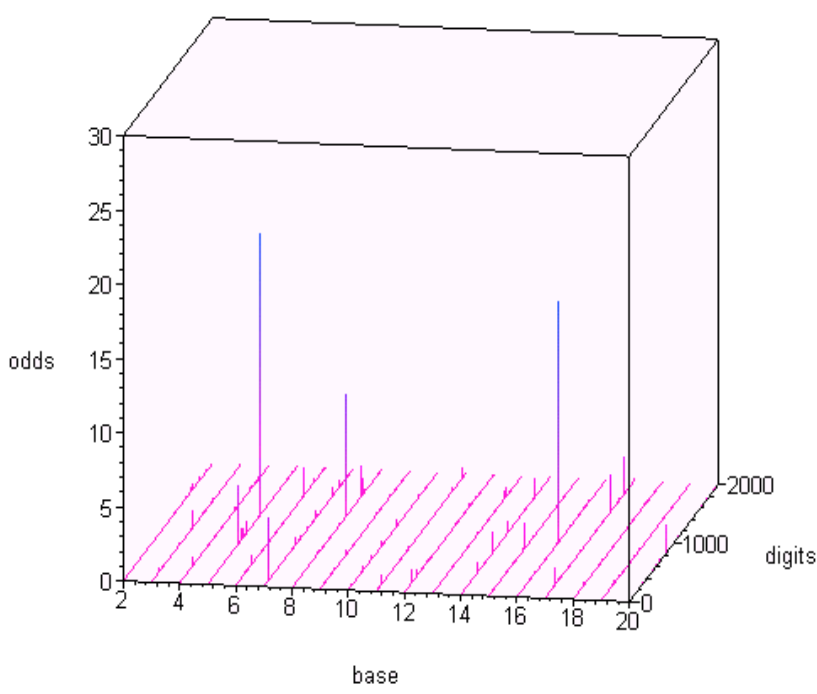

Figure 1. Sequences of identical digits in the transcendental number $e$ expressed in different bases between base 2 and base 20, and with up to 2000 digits in the numerical expansion

For comparison, Figure 2 shows the corresponding results for completely random sequences of digits for the different bases. We see that Figure 1 and Figure 2 are essentially similar. There are a few instances of somewhat longer sequences of identical digits with odds of the order of 5 to 25 scattered over an undergrowth of odds of the order of just a few units.

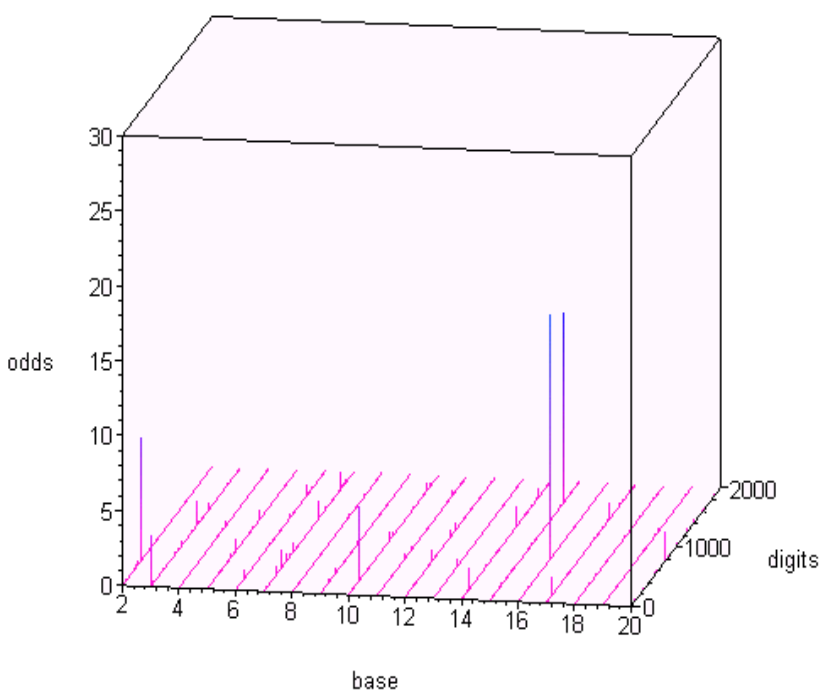

Figure 2. As in Figure 1 and in the same scale, but for comparison for sequences of random digits, giving a distribution with essentially the same structure

\section{Representation of $\pi$ as Function of Base and Depth}

Turning now to the transcendental number $\pi$, Figure 3 shows a plot of $2 \pi$ in the same manner and the same scale. Comparing this figure with the previous Figure 1 and Figure 2, we note that the strings in the transcendental number $2 \pi$ are radically different. The undergrowth is less prominent, and there are no instances with odds of the order of 5 to 25 units.

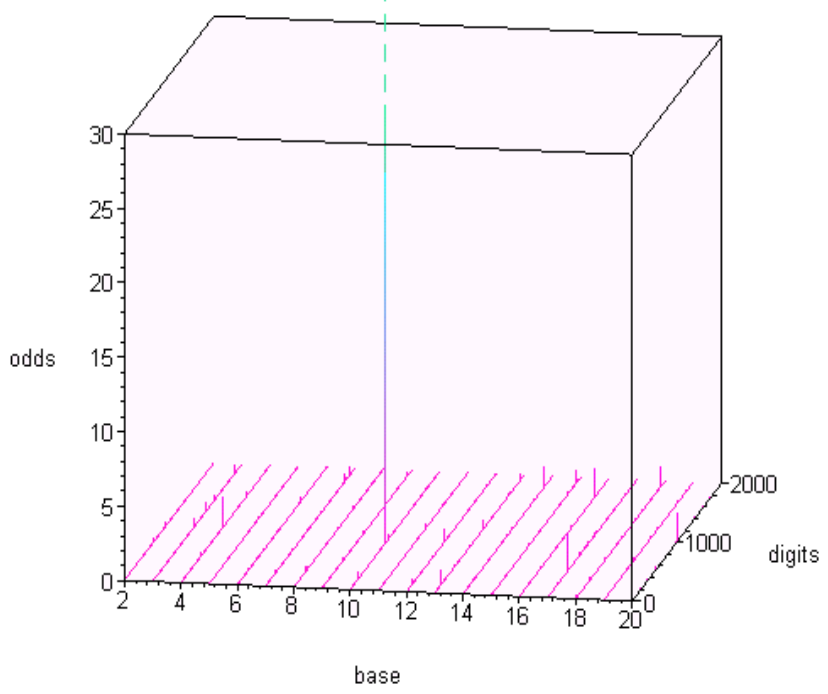

Figure 3. Same as Figure 1 and Figure 2 and in the same scale but for $\pi$, showing less pronounced occurrences of sequences with identical digits, except for the off-scale case of base ten at $3 \times 256$ digits given in Table 1

Instead there is now one huge peak at depth $768=3 \times 2^{8}$ for base ten. It seems as if all the scattered higher odds are now combined into one prominent marker. Increasing the vertical scale a hundred times as in Figure 4, we can see 
just how spectacular this marker at base ten really is. This marker completely overshadows all other strings in the number. This would then be regarded as a strong candidate for a marker to a message hidden in the transcendental number $2 \pi$.

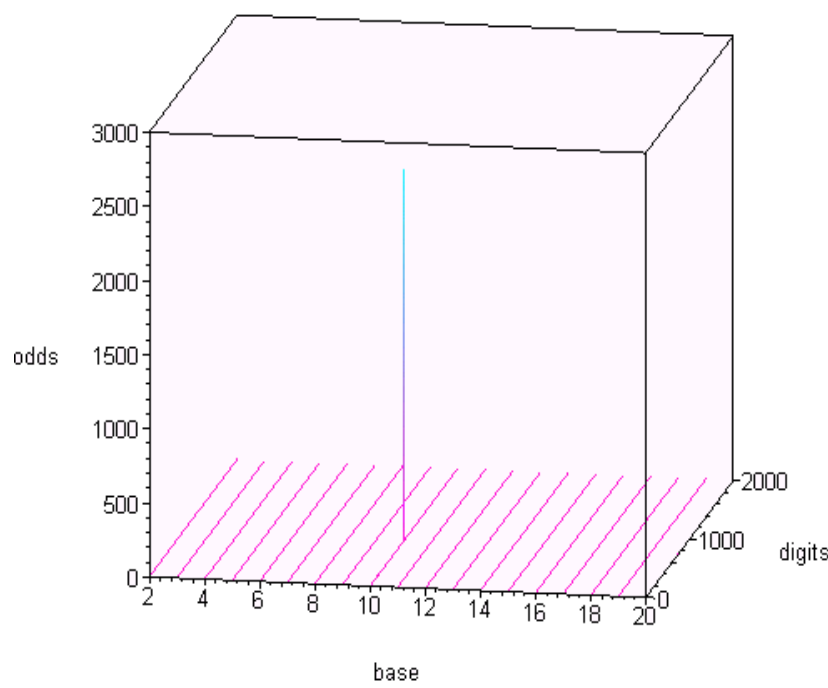

Figure 4. Same as Figure 3 but with vertical scale extended by a factor 100 , showing the full height of the sequence of identical digits for base ten at $3 \times 256$ digits

Table 1 shows the number $2 \pi$ calculated with 768 digits in base ten ( $c f$ also, $e g$, [6]), where we clearly see the marker consisting of a sequence of seven zeros (actually a sequence of seven nines rounded off).

Table 1. First $3 \times 256=768$ digits in $2 \pi$ for base 10 showing the sequence of seven zeros at the end (actually the sequence ... 2699999997 ... rounded off)

6.28318530717958647692528676655900576839433879875

021164194988918461563281257241799725606965068423

413596429617302656461329418768921910116446345071

881625696223490056820540387704221111928924589790

986076392885762195133186689225695129646757356633

054240381829129713384692069722090865329664268721

452049828254744917401321263117634976304184192562

850818343072873578518072002266106109764093304276

829390388302321886611454073151918390618437223476

386522358621023709614892475992549913470377150544

978245587636602389825966734672488131328617204278

989279044947438140435972188740554107843435258635

350476934963693533881026400113625429051712165557

154268551557921834727435744293688180244990686029

309917074210158455937851784708403991222425804392

172806883631962725954954261992103741442270000000

\section{Concluding Remarks}

The above study thus shows a remarkable occurrence of a string of seven zeros in the representation of $2 \pi$ (or $\pi$ ) if expressed in $3 \times 2^{8}$ digits for base ten, and which does not even remotely occur for this particular transcendental number expressed in any other base, nor for any other commonly occurring transcendental number in physics expressed in similar range of bases and digits.

This may thus be another possible anthropic occurrence in nature of somewhat the same kind as the fine-tuning of physical constants in the universe seemingly necessary to permit life to exist and be able to observe it [7]. Thus, assuming for the sake of argument that this marker does indeed point to a message buried inside a digital representation of $\pi$, what could be the possible purpose if any - of such a message?

Is it perhaps a marker to a picture of the creator of the Universe - put there at an unobtrusive place in his creation, like when a human artist has finished his painting or sculpture and signs it with his name? Or when a human builder writes his name in the wet concrete of the staircase he has just finished? Or like when humans sent their first space probe towards the stars and attached a plaque with a picture displaying a man and a woman? Or maybe is it like the prehistoric human cave paintings depicting animals to be hunted?

Or is it perhaps just some graffiti, put there with no meaning nor purpose whatsoever, other than maybe to please an exhibitionistic urge of its maker?

While I have been writing this on my PC, a tiny $1 \mathrm{~mm}$ spider has been spinning a web invisible to me over a corner of my computer screen. She is now patiently sitting there, hoping to catch some tiny prey lured by the light of the screen, whatever small prey that might be. We have together just listened to Sissel singing Silent Night, and I am now trying to summarise my thoughts on the subject I have been discussing above. But maybe any attempt to understand those matters is as hopeless as it must be for this little spider to comprehend the meaning and purpose of the symbols appearing on the screen underneath her and the strange sounds she has just heard...

\section{References}

[1] C. Sagan, Contact, (Century Hutchinson Ltd, 1986).

[2] H. S. M. Coxeter, Non-Euclidean Geometry (Univ. Toronto Press 1942, reissued 1998 by Mathematical Assoc. of America).

[3] http://en.wikipedia.org/wiki/ Elliptic_geometry retrieved 2014-1102.

[4] http://en.wikipedia.org/wiki/Hyperbolic_geometry retrieved 201411-02.

[5] M. B. Monagan, K. O. Geddes, K. M. Heal, G. Labahn, S. M. Vorkoetter, J. McCarron, P. DeMarco, Maple 7 Programming Guide (Waterloo Maple, 2001).

[6] http://www.exploratorium.edu/pi/pi_archive/Pi10-6.html http://www.piday.org/million/ retrieved 2014-11-02.

[7] U.-G. Meissner et al., "Life on earth - An accident? Chiral symmetry and the anthropic principle”, International Journal of Modern Physics E 23, 1461005 (2014). 